\title{
Maternal Dominance, Maternal Condition, and Offspring Sex Ratio in Ungulate Mammals
}

\author{
Ben C. Sheldon ${ }^{1, \star}$ and Stuart A. West ${ }^{2, \dagger}$ \\ 1. Edward Grey Institute, Department of Zoology, University of \\ Oxford, South Parks Road, Oxford OX1 3PS, United Kingdom; \\ 2. Institute of Cell, Animal, and Population Biology, University of \\ Edinburgh, Edinburgh EH9 3JT, United Kingdom
}

Submitted March 18, 2003; Accepted August 5, 2003;

Electronically published January 14, 2004

Online enhancements: appendix tables, literature cited.

AвStract: Trivers and Willard's suggestion that natural selection favors maternal control of offspring sex ratio in relation to maternal condition has been much debated. The theoretical plausibility of the idea, under some conditions, is firmly established, and there is strong empirical support for conditional sex allocation in some taxa. However, the extent to which this hypothesis can be applied to mammals, particularly ungulates, has been more controversial. We used metaanalysis to review published studies of the Trivers-Willard hypothesis within ungulates and to assess the overall level of empirical support for the hypothesis. Overall, data from 37 studies of 18 species suggested a weak but significant positive correlation between maternal condition and sex ratio $(r=+0.09)$. However, average effect size differed markedly between different categories of studies. Studies using measures of maternal condition that were taken preconception and on the basis of behavioral dominance provided strong evidence for a relationship between maternal condition and the sex ratio $(r=+0.17-0.25)$. In contrast, studies that used morphological or physiological measures of condition that were measured postconception provided little or no evidence for a relationship between maternal condition and sex ratio $(r=+0.05-0.06)$. There are several reasons to suggest that data collected postconception and relying on morphological measures of condition are less likely to capture variables that cause selection for biased sex allocation. In addition, we found that the relationship between sex ratio and maternal condition depended on life-history characteristics; relationships were stronger when sexual size dimorphism was more male biased and when gestation periods were longer. Overall, our analyses suggest that data from ungulates are consistent with the Trivers-Willard hypothesis but only when appropriate measures are used.

\footnotetext{
* Corresponding author; e-mail: ben.sheldon@zoology.oxford.ac.uk.

† E-mail: stu.west@ed.ac.uk.
}

Am. Nat. 2004. Vol. 163, pp. 40-54. (c) 2004 by The University of Chicago. 0003-0147/2004/16301-30102\$15.00. All rights reserved.
Keywords: comparative analysis, meta-analysis, parental investment, sex allocation, sexual selection.

In cases where the reproductive value of male and female offspring is affected differently by an environmental variable, parents may be selected to adjust the ratio of allocation of resources to male and female offspring (Charnov 1982). The theoretical and empirical study of this phenomenon, sex allocation, has been very successful in some taxa, for example, in parasitoid wasps, eusocial hymenoptera, and hermaphrodites (Charnov 1982; Godfray 1994; Bourke and Franks 1995; West et al. 2000). In many of these taxa, empirical data show a good fit to the predictions of simple models. In contrast, the degree to which empirical data support theoretical models in some other taxa has been much debated (Williams 1979; CluttonBrock and Iason 1986; Hewison and Gaillard 1999; Palmer 2000; Brown 2001; Brown and Silk 2002; West and Sheldon 2002).

One of the most debated issues concerns the empirical support for one of the earliest models of individual sex allocation. Trivers and Willard (1973) suggested that in mammals where maternal condition affected the fitness of offspring and where the fitness of male offspring was more strongly affected than the fitness of female offspring, females in good condition should bias their offspring sex ratio in favor of males because this would be the sex that yielded the highest marginal fitness returns. Although this was not formally modeled in the original article, this is a special case of a more general formulation of the effect that variables with sex-differential effects on offspring fitness select for biased sex allocation (Charnov 1979; 1982). Consequently, there is already strong support for the underlying logic of the Trivers-Willard hypothesis from numerous other situations, such as the occurrence of sex ratio adjustment by female solitary parasitoids in response to host size (Godfray 1994; West and Sheldon 2002).

A particular difficulty in testing the Trivers-Willard hypothesis in mammals is that subsequent theoretical work has shown that the expected sex ratio skew in relation to maternal condition may, in fact, not be predictable. Leimar 
(1996) modeled the Trivers-Willard hypothesis using reproductive value of offspring rather than their immediate reproductive success and showed that the predicted relationship between maternal condition and offspring sex ratio could be positive or negative. This complication arises because of the possibility of strong maternal transmission of condition to daughters, such as through inheritance of territories or social rank. When this occurs, it provides a benefit to females in good condition who produce daughters because these daughters can themselves produce high-quality daughters. Consequently, the predicted direction and strength of any relationship between maternal condition and offspring sex ratio will depend on the relative importance of maternal effects on daughters and sexual selection on sons. Leimar's (1996) analysis illustrates a general problem with tests of sex allocation theory, which applies to many taxa; there are very few empirical data available to allow clear a priori prediction about the direction of effects (West and Sheldon 2002). In the case of mammals, too little is known about the details of life-history variation in males and females to make a firm prediction about the direction of sex ratio skews expected in any but the very best studied species, and in that sense, most empirical data cannot really be said to support or not support Trivers and Willard's theory.

A second, unrelated area of disagreement concerns whether there is a common pattern in studies of sex ratio variation in relation to maternal condition. Many studies have been published as tests of the Trivers-Willard hypothesis, but in only some of these studies have significant associations between a measure of maternal condition and sex ratio been reported. The apparent variability has led to suggestions that there is no evidence that sex ratios are related to maternal condition and that those studies revealing relationships may represent a biased sample of work carried out (Clutton-Brock and Iason 1986; FestaBianchet 1996; Hewison and Gaillard 1999; Palmer 2000). However, there are several difficulties with this conclusion. First, nonquantitative statements about the overall pattern of research in a field may be unreliable because a set of results in one or other direction can almost always be selected from a large field of published work (Arnqvist and Wooster 1995). Second, some statements about variability of results in studies of sex ratios have mistakenly concentrated on population sex ratios, concluding that there is little evidence that sex ratio variance between populations is anything other than binomial (e.g., Palmer 2000). However, the distribution of population sex ratios has little relevance to the question of whether individuals within populations adjust their sex ratio because models of individual sex allocation are based on how variance in the sex ratio is distributed within, not between, populations (West and Sheldon 2002). An alternative approach has been to assess whether the variance in sex ratios within populations differs from that expected from a binomial distribution (e.g., Williams 1979). However, tests of variance have rather little power, and in addition, the assumption that failure to reject the null hypothesis of binomial sex ratio variance (at either population or family level) is indicative of the absence of sex ratio adjustment is questionable. Separate processes may lead to sex ratio variance being greater than or less than binomial. For example, if the probability of being one sex varies consistently across positions within a sequence of births, subbinomial variance may result (Krackow et al. 2002). In contrast, if families born under particular conditions tend to be composed of one sex, then greater than binomial variance may result. Both sorts of process are known to occur in birds and mammals (e.g., Austad and Sunquist 1986; Huck et al. 1990; Badyaev et al. 2002; West and Sheldon 2002). Consequently, it is hard to know what distribution of sex ratio variances to expect across species. Finally, few authors have tried to explain differences in the outcome of studies based on the quality of data collected. As we show, this is important for understanding variation in the results of studies of sex ratio in relation to maternal condition.

Our aim in this article is to conduct a quantitative assessment of the evidence that maternal condition (defined in various ways) is associated with offspring sex ratio. In addition, we aimed to explore possible sources of variation in the relationship between offspring sex ratio and maternal condition. We restricted our analysis to studies of ungulate mammals for four reasons. First, this group has been the subject of much work in this area, and by restricting our analysis to a relatively well-defined taxonomic group, we hoped to control for some of the possible sources of variation between studies. Second, extensive work on ecology and life history in ungulates means that data are more likely to be available to test explanations for variation in effect size. Third, many ungulates have small litters; when litters are large, litter size and sex ratio may interact in unpredictable ways (Williams 1979; Frank 1990). Finally, many ungulates have polygynous mating systems and are sexually size dimorphic, and male reproductive success is settled by the outcome of male-male competition. These characteristics suggest that ungulates would be a particularly appropriate model for the TriversWillard hypothesis (Hewison and Gaillard 1999). We used meta-analytic techniques to compare studies and draw overall conclusions about the pattern of results. Metaanalysis is a technique for combining information from different studies and deriving quantitative statements about the patterns shown by the studies as a whole. Further details about the methods involved can be found in articles 
by Rosenthal (1991), Arnqvist and Wooster (1995), Gurevitch and Hedges (1999), and Rosenberg et al. (2000).

We do not consider that most data concerning sex ratio variation and maternal condition in ungulates can be treated as a formal test of the Trivers-Willard hypothesis because of the general paucity of data needed to predict whether, and in what direction, any sex ratio adjustment is expected. However, given that such data are very difficult to obtain, there are several valid secondary questions such as asking what pattern is found across studies and whether variation between studies can be explained.

\section{Hypotheses Tested}

We aimed to answer the following specific questions, relating to both the overall pattern of results across studies and the source of differences between studies. First, what is the overall relationship between offspring sex ratio and measures of maternal condition across studies in ungulates?

Second, is the relationship between sex ratio and maternal condition dependent on the type of measure of maternal condition employed? We tested two differences in the way that maternal condition had been measured. One concerned whether the measure used involved a morphological (or physiological) measure of condition (e.g., body mass, a measure of fat load, whether an individual was lactating at the time of conception) or, alternatively, a measure of behavioral dominance. While dominance is not a measure of condition, the use of condition in the context of the Trivers-Willard hypothesis is just to stand for maternal ability to invest in offspring, which may clearly be related to dominance. The second factor that we tested concerned the time at which maternal condition was determined relative to conception. Many of the studies included report the association between the sex ratio and a measure of maternal condition for females shot by hunters or for culling purposes, and these measures are by definition postconception; in other studies, maternal condition was determined before conception occurred.

Third, is the relationship between sex ratio and maternal condition influenced by population management status? Kruuk et al. (1999) showed that population density influenced the relationship between maternal dominance and sex ratio in red deer Cervus elaphus and suggested that differences between studies could be explained by management status, with a relationship between sex ratio and dominance expected only at low densities. Density could not always be determined for the study populations, so we classified population management status into three categories (captive, semiwild, or free ranging) on the basis of subjective classifications of the descriptions of the study populations.
Finally, does the relationship between sex ratio and maternal condition depend on variation in life-history characteristics across species? For example, Kojola (1997a) suggested that effects of maternal condition would be more pronounced in ungulates with small litters. Alternatively, large litters might facilitate sex ratio adjustment postimplantation because the sacrifice of one or two embryos would represent a smaller loss of potential reproductive success. In addition, we tested for the effect of gestation time, sexual size dimorphism, and social organization because all might influence selection on sex ratio adjustment. For example, Hewison and Gaillard suggested that TriversWillard effects might be more marked in species with more marked sexual selection on males because this should increase the fitness return from sex-biased investment. Hence, if this hypothesis is correct, we expect to see stronger effects when sexual size dimorphism is more male biased. West and Sheldon (2002) suggested that an important determinant of the strength of sex allocation biases would be the degree to which the environment offspring would inhabit could be predicted at the time allocation decisions were made. Hence, all else being equal, weaker effects would be associated with longer gestation periods.

\section{Data Collection}

We collected studies of the Trivers and Willard hypothesis by performing literature searches using the ISI Web of Science; all articles published up to January 31, 2003, were considered. We forward-searched by identifying all articles that had cited three major early studies of sex ratio variation in mammal by Trivers and Willard (1973), CluttonBrock et al. (1984), and Clutton-Brock and Iason (1986). All articles that were judged from the abstract to have potential relevance to sex ratio variation in ungulates were then consulted, and the articles that had in turn been cited by these articles were consulted. While performing this procedure, we adopted broad definitions of maternal condition and sex ratio in order to maximize the number of studies screened.

\section{Inclusion of Studies}

We located 73 studies that were potentially related to the Trivers and Willard hypothesis in ungulates; a full list appears in appendix B in the online edition of the American Naturalist. However, only a proportion of these actually contained data that could be used in a meta-analysis of the hypothesis. Some studies were excluded because they did not report sufficient information to extract for a metaanalysis (at minimum, a test statistic or $P$ value and a sample size were needed). Several studies were presented as tests of the Trivers and Willard hypothesis when in fact 
they were not. For example, we found a number of articles that reported the correlation across populations between average maternal condition and average sex ratio (e.g., Verme 1983; Skogland 1986) or the difference in sex ratio between groups of deer maintained in captivity on different diets (Verme 1985) or the difference in mean sex ratio between two wild populations subject to different management practices (e.g., Smith et al. 1996). Conditional sex allocation, of the kind proposed by Trivers and Willard, concerns the decisions that individuals within a population make, and comparisons of average behavior between different populations are not of obvious relevance to this question. For example, size-advantage models of sex allocation in parasitoid wasps have the same underlying logic as the Trivers and Willard model and have overwhelming empirical support (West and Sheldon 2002). By exposing ovipositing females of the parasitoid Lariophagus to different distributions of host size, Charnov et al. (1981) showed experimentally that it is the relative distribution of resources experienced by individuals within a population that influences sex allocation decisions, not the absolute size of the resource across populations. It is possible that any mechanism individuals use to adjust sex ratio within populations might be influenced by betweenpopulation differences (e.g., in food availability). However, there seems to be no clear means to decide whether this is the case. Consequently, while differences in mean sex ratio between populations correlated with resource availability are interesting and require explanation, they do not reveal whether individuals within a population adjust their sex ratio in relation to their condition. Facultative sex allocation leads to evolutionarily stable strategy (ESS) sex allocation ratios that differ from equality, but it is very hard to predict where the new ESS lies (Frank 1990).

A second reason for the exclusion of some studies concerned the way in which behavioral dominance was defined. Some studies (e.g., Clutton-Brock et al. 1984; Côté and Festa-Bianchet 2001; S. D. Côté, personal communication) defined it relative to the entire population that an individual interacted with, whereas others (e.g., Clutton-Brock et al. 1986; Festa-Bianchet 1991) derived cohort-specific measures that control for any influence of age. If we were interested specifically in how dominance related to the sex ratio of offspring, the latter sort of measure would be the appropriate one to use. However, in the context of the Trivers-Willard hypothesis, dominance is used simply as an index of the extent to which mothers can invest in their offspring, and whether dominance status is partly related to age is unimportant (see Hewison et al. 2002 for similar arguments in relation to the effect of age on sex ratio). Because an individual competes for resources with all members of its population, not just those within its cohort, it is an individual's ability to compete relative to the rest of the population that should determine how much it can invest in offspring. In some cases, the two measures of dominance give broadly similar results in terms of their correlation with the sex ratio (e.g., Clutton-Brock et al. 1984: $r=0.26$ with the overall dominance measure; Clutton-Brock et al. 1986: $r=0.44$ with the age-corrected measure), but in other cases, controlling for age makes a large difference because dominance is very strongly related to age (e.g., Côté and Festa-Bianchet 2001; S. D. Côté, personal communication). Consequently, we excluded two studies that reported relationships between sex ratio and age-corrected dominance but did not report the correlation with the overall dominance measure (Clutton-Brock et al. 1986; Festa-Bianchet 1991). Further details are given in appendix $\mathrm{B}$, which contains an explanation for the noninclusion of each of the remaining studies that were consulted.

Data on life-history characteristics of the species of ungulates included in the analysis were obtained from a variety of sources, including the articles reporting the sex ratio effect (typically for litter size). Sexual size dimorphism was defined as the residual from a regression of log female body mass on log male body mass (Ranta et al. 1994). The life-history data used in the analysis, together with additional sources for this data, are given in appendix $\mathrm{C}$ in the online edition of the American Naturalist.

\section{Data Analysis}

From each article, we extracted the relevant statistical information relating to the relationship between the sex ratio and maternal condition (see table 1 for a list of articles, and see table A1 in the online edition of the American Naturalist for full details of the information extracted from each study). Several different types of test were performed by the original articles including those that compared mean condition between females with male or female offspring, and those that tested for a correlation between sex ratio and maternal condition. Some tests reported this information for a single reproductive event by each female (e.g., those that relied on postmortem measures of condition and sex ratio), while others (e.g., Clutton-Brock et al. 1984) measured the sex ratio over each female's reproductive lifespan. There was also variation between studies in the time at which the sex ratio was measured, ranging from the blastocyst stage (Flint et al. 1997) to postpartum. The statistical methods used to test for the relationship, the type of sex ratio measure used, and the time during development at which the sex ratio was measured could all potentially influence the ability to detect a relationship between maternal condition and sex ratio, but we made no attempt to control for these possible sources of vari- 
Table 1: Ungulate taxa and studies used in meta-analysis

\begin{tabular}{ll}
\hline Taxon & \multicolumn{1}{c}{ Studies included in meta-analysis } \\
\hline Fallow deer Dama dama & Birgersson 1998 \\
Roe deer Capreolus capreolus & Wauters et al. 1995; Hewison and Gaillard 1996; Hewison et al. 1999 \\
White-tailed deer Odocoileus virginianus & Burke and Birch 1995 \\
Red deer Cervus elaphus & Clutton-Brock et al. 1984; Flint et al. 1997; Kohlmann 1999 \\
Reeve's muntjac Muntiacus reevsi & Chapman et al. 1997 \\
Moose Alces alces & Schwartz and Hundertmark 1993; Nygén and Kojola 1997 \\
Reindeer Rangifer tarandus & Kojola and Eloranta 1989; Kojola 1993; Kojola and Helle 1994; Reimers \\
& and Lenvik 1997; Reimers 1999; Weladji et al. 2003 \\
Cape mountain zebra Equus equus zebra & Lloyd and Rasa 1989 \\
Bighorn sheep Ovis canadensis & Hass 1991 \\
Arrui Ammotragus lervia & Cassinello 1996; Cassinello and Gomendio 1996 \\
Bison Bison bison & Rutberg 1986; Wolff 1988, 1998; Shaw and Carter 1989 \\
Cuvier's gazelle Gazella cuvieri & Alados and Escos 1994 \\
Pronghorn Antilocapra americana & Byers 1997 \\
Domestic horse Equus caballus & Monard et al. 1997; Cameron et al. 1999 \\
Domestic pig Sus scrofa domesticus & Mendl et al. 1995; Meikle et al. 1996 \\
Wild boar Sus scrofa & Fernandez-Llario et al. 1999 \\
Mountain goat Oreamnos americanus & Côté and Festa-Bianchet 2001 \\
Soay sheep Ovis aries & Lindström et al. 2002 \\
Mouflon Ovis musimon & Llandete-Castillejos et al. 2001 \\
\hline Note: Full details of the test statistics, sample sizes, and effect sizes are given in appendix A in the online edition of the American Naturalist.
\end{tabular}

ation in effect size, in part because the number of studies available was not very large.

Rather than reporting the relationship between individual condition and the sex ratio, several studies reported data as a test of Williams's (1979) hypothesis that litter size and sex ratio should be jointly optimized in species with litters greater than one (e.g., Burke and Birch 1995; Cassinello and Gomendio 1996). For example, one might predict that in a species where litter size can be one or two, those females in best condition should produce two sons and those in slightly less good condition should produce one son and one daughter, then two daughters, and then a son, while the reproductive group in poorest condition should produce daughters. This hypothesis requires an assumption about the relative cost of male and female offspring in order to know how to order the different combinations of litter sizes and sexes (Frank 1990, 1998). Consequently, it is hard to assess whether data fit the expectation of Williams's (1979) hypothesis. We extracted data relating the sex ratio alone to maternal condition from these studies as follows (see also fig. 1 for a hypothetical example). Because all of the studies that tested Williams's (1979) hypothesis reported test statistics that compared different litter size/sex ratio combinations without directional contrasts tested between groups, we combined this information with the correspondence between the observed and expected rank ordering of the means between groups if sex ratio were the only variable related to maternal condition (thus, a litter of one containing one fe- male had the same expected rank ordering as a litter of two containing two females or a litter of three containing three females) using Rice and Gaines's (1994) ordered heterogeneity test. This process will not yield exactly the same effect size as would a correlation between sex ratio and maternal condition, but we chose this procedure as preferable to discarding these studies.

We used standard methods (Rosenthal 1991; Rosenberg et al. 2000) to convert the reported test statistics into a common measure of effect size, the correlation coefficient $(r)$. Effect sizes were $z$ transformed before analysis to correct for asymptotic behavior of $r$, and in all analyses, individual effect sizes were weighted by the inverse of the variance associated with the effect size estimate (Rosenberg et al. 2000). We used random effect models in all of the meta-analyses performed here. Meta-analyses can be performed using either fixed or random effects models, but there are several reasons to suspect that random effect models are more appropriate for analysis of typical ecological data (Gurevitch and Hedges 1999). Random effect models allow for two sources of error in effect size simultaneously: that because of sampling error and that because of real differences in effect size between studies in a sample. Because there is no reason to assume that all ungulate mammals would be subject to the same factors shaping the relationship between the sex ratio and maternal condition and because there were differences between studies in the way condition and the sex ratio were measured, the studies in this sample are unlikely to share 


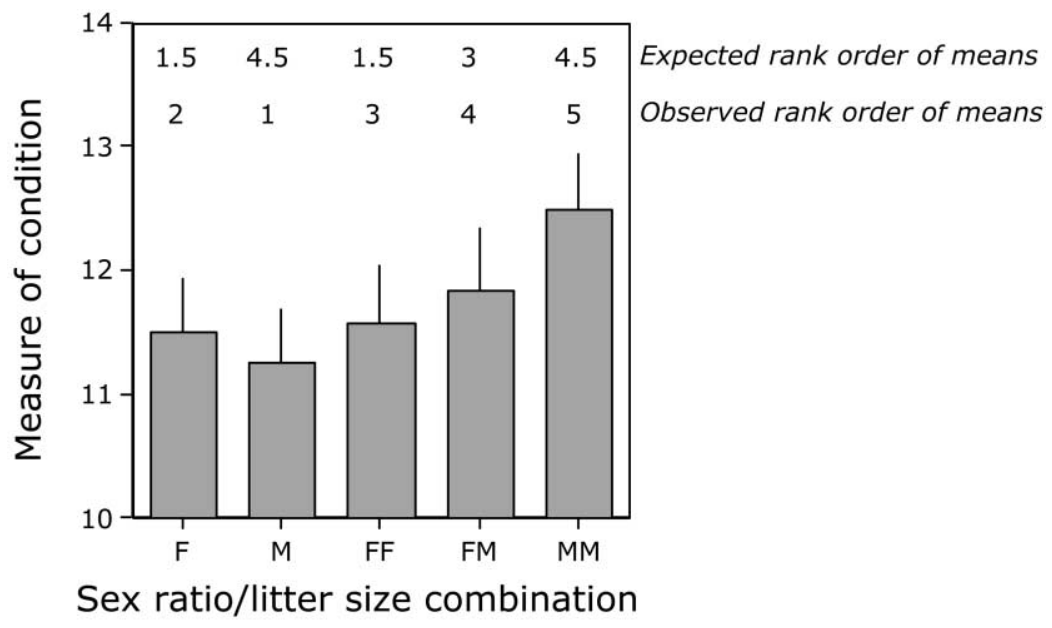

Figure 1: Hypothetical example of conversion of a test of Williams's (1979) hypothesis about joint optimization of litter size and sex ratio to a directional statistic relating sex ratio to maternal condition. Overall test of association between maternal condition and sex ratio/litter size combination in this hypothetical example: $F=1.21, \mathrm{df}=4,32, P=.33$. The correlation between the observed ordering of the means and that expected if maternal conditions were related to sex ratio alone is, in this case, $r_{\mathrm{S}}=0.1581$. Hence, following Rice and Gaines (1994), an ordered heterogeneity test of whether sex ratio is related to maternal condition can be derived from the test statistic: $r_{\mathrm{s}} P_{\mathrm{c}}=0.1581 \times 0.67=0.106$, with $k=5$, yields $P=.64$ (two-tailed).

a common effect size. Because the distribution of effect sizes is not known, we used bootstrapping to obtain confidence intervals around estimates of mean effect size, and $P$ values for most statistical tests were obtained by randomization $\left(9,999\right.$ resampling events; denoted by $\left.P_{\mathrm{r}}\right)$. At present, meta-analysis is restricted to univariate analyses of factors that might explain differences in effect size. All analyses were conducted using MetaWin 2.0 (Rosenberg et al. 2000).

\section{Results}

Our literature search located 73 published studies that we examined for reported associations between some measure of condition and offspring sex ratio. Of these, we were able to extract data for the meta-analysis from 40 , of which one (Meikle et al. 1993) was discarded because it consisted of a subsample of a subsequent study (Meikle et al. 1996), and two were discarded because they reported effects of dominance on sex ratio correcting for age effects on dominance (Clutton-Brock et al. 1986; Festa-Bianchet 1991). The final sample analyzed contained 37 studies of 18 species (table A1). In the majority of the other cases, the study did not report any information about condition and the sex ratio, it provided insufficient details to allow calculation of an effect size, or it was not a test of the TriversWillard hypothesis. Appendix B lists all of the studies consulted and the reason for their exclusion from the meta-analysis.

\section{Overall Effects}

Within the subset of studies that were used, the estimated mean effect size was $r=0.090$ (95\% confidence interval $[\mathrm{CI}]: 0.041-0.144, n=37$ ), suggesting a weak, but statistically significant, mean effect size for the relationship between offspring sex ratio and condition. However, there was evidence of significant heterogeneity between studies $\left(\chi^{2}=51.48, \mathrm{df}=36, P=.046\right)$, indicating that, overall, the studies were not drawn from a homogeneous group. We therefore tested two potential sources of heterogeneity: the type of study conducted and the species on which it was conducted. Both factors might potentially influence the strength of any relationship between the sex ratio and maternal condition

\section{Differences between Studies}

Multiple studies were available for nine of the 18 species in the sample (table A1; domestic pig and wild boar were treated as the same species). Average effect size did not differ significantly between species in the sample, although this result was marginally nonsignificant $\left(\chi^{2}=18.99\right.$, $\left.\mathrm{df}=8, P_{\mathrm{r}}=.09, n=9\right)$. Mean effect sizes were statistically significant (and positive) for red deer, arrui, bison and feral horses. In contrast to the effect of species, mean effect size was influenced by the type of study $\left(\chi^{2}=\right.$ $\left.8.55, \mathrm{df}=1, P_{\mathrm{r}}=.009\right)$, with the mean effect size for studies that were conducted using measures of behavioral dominance approximately four times that of studies that 
had used morphological condition measures (table 2; fig. $2 a)$.

These conclusions were further strengthened when they were repeated excluding a single study (Mendl et al. 1995) that may not provide a very appropriate test of whether there is a relationship between sex ratio and maternal dominance (table A1; see Meikle et al. 1997; Mendl et al. 1997 for further discussion). The overall mean effect size was $r=0.087$ (95\% CI: $0.041-0.141, n=36$ ), and the difference between the two classes of studies was larger $\left(\chi^{2}=12.79, \mathrm{df}=1, P_{\mathrm{r}}=.002\right)$. Exclusion of this study suggested that species with replicate studies did yield significantly different mean effect sizes, although the effect was marginally nonsignificant $\left(\chi^{2}=24.33, \mathrm{df}=8\right.$, $\left.P_{\mathrm{r}}=.054, n=9\right)$.

A second potential source of difference in effect size concerns the time at which maternal condition was measured relative to conception. Postconception measures of condition might mask any association between maternal condition and conception sex ratio, particularly if there is sex-biased investment before birth. The timing of maternal condition relative to conception could be determined from the original sources for 34 of 37 studies (92\%); of these, two of 34 collected data continuously (i.e., both before and after conception). Of the remainder, 15 of 32 (47\%) used a measure taken either before or at conception (table A1). When all studies were included, timing of measurement of maternal condition was unrelated to mean effect size $\left(\chi^{2}=6.84, \mathrm{df}=3, P_{\mathrm{r}}=.15\right)$. When we excluded the two classes for which timing was unknown and data had been collected continuously, a significant difference in mean effect size depending on the timing of maternal condition measure was revealed $\left(\chi^{2}=6.32, \mathrm{df}=1\right.$, $\left.P_{\mathrm{r}}=.026\right)$. Mean effect size was four times as large when condition was measured at or before conception, compared with after conception (table 2; fig. $2 b$ ). Hence, these analyses suggest that timing of measurement might influence the effect size obtained by a study.

Studies of the Trivers-Willard effect have been conducted under a range of conditions, from completely wild, unmanaged populations to completely captive populations. Kruuk et al. (1999) showed that population density influenced the relationship between maternal condition and sex ratio in red deer and suggested that Trivers-Willard effects might be more likely to be detected in populations kept below the carrying capacity. Because we were unable to obtain data on population management status for all of the studies here, we tested whether wild and captive populations differed with respect to mean effect size; we found no evidence that this was the case $\left(\chi^{2}=0.59\right.$, $\mathrm{df}=1, P_{\mathrm{r}}=.48$; table 2 ).

\section{Mutually Confounded Variables}

Meta-analytic software that can test the effects of multiple moderator variables simultaneously has not yet been developed; therefore, we were unable to test which of the moderator variables was most influential. We asked whether any of our moderator variables were confounded with each other because this would affect our ability to determine their relative influence on effect size. There was no relationship between whether a study used a morphological or behavioral measure of condition and the timing of that measure relative to conception, excluding those studies for which timing could not be determined $\left(\chi^{2}=1.05, \mathrm{df}=1, P=.31, n=32\right)$ or between the timing of the measure of condition and population status $\left(\chi^{2}=0.85, \mathrm{df}=1, P=.36, n=32\right)$. Hence, the influence of the type of condition measure and the time at which condition was measured should be independent of one another. There was a tendency for studies of wild populations to be more likely to use morphological mea-

Table 2: Summary statistics of effect sizes in studies of sex ratio in relation to maternal condition in different categories of study

\begin{tabular}{|c|c|c|c|}
\hline Category of study & $\begin{array}{c}\text { Mean effect size } \\
(r)\end{array}$ & $N$ & $95 \% \mathrm{CI}$ \\
\hline \multicolumn{4}{|c|}{ Type of measure of maternal condition: } \\
\hline Morphological & .059 & 26 & $.016-.108$ \\
\hline Behavioral & .246 & 11 & $.075-.375$ \\
\hline Behavioral $^{a}$ & .287 & 10 & $.159-.401$ \\
\hline \multicolumn{4}{|c|}{ Timing of condition assay relative to conception: } \\
\hline Preconception & .167 & 15 & $.088-.272$ \\
\hline Postconception & .045 & 17 & $-.010-.105$ \\
\hline \multicolumn{4}{|l|}{ Population status: } \\
\hline Wild & .090 & 26 & $.040-.150$ \\
\hline Semiwild & .020 & 2 & $-.043-.085$ \\
\hline Captive & .148 & 9 & $-.019-.343$ \\
\hline
\end{tabular}

a Excluding Mendl et al. (1995); see appendix A in the online edition of the American Naturalist for details. 

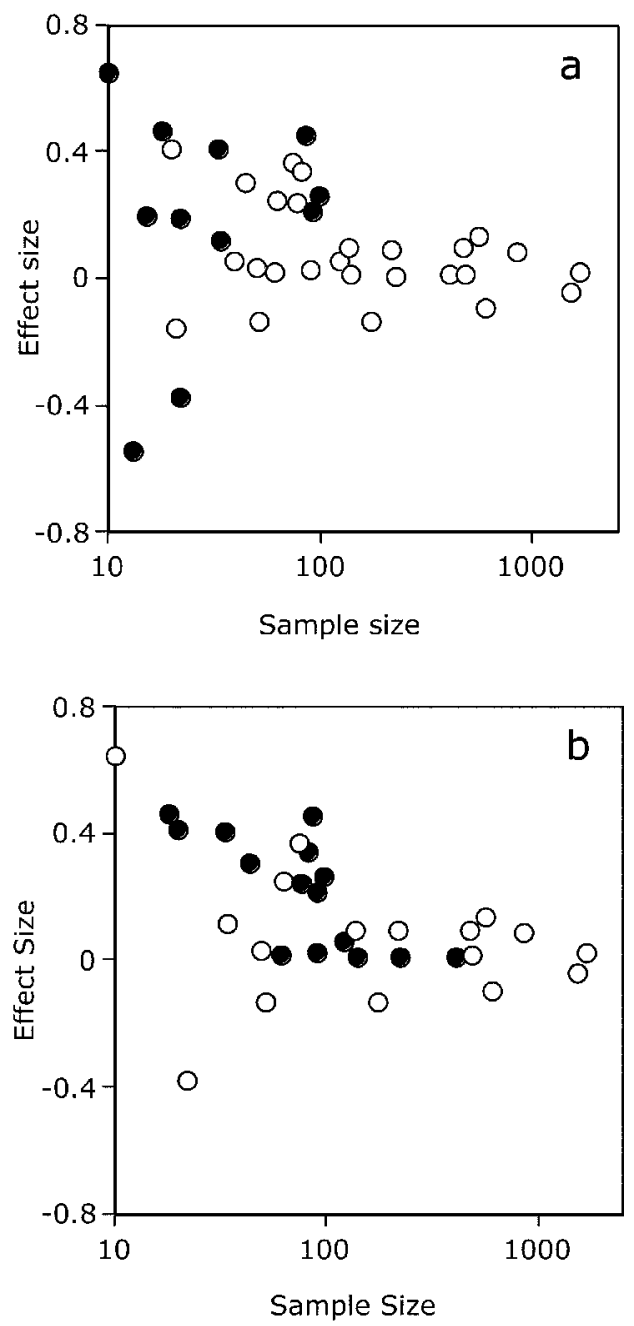

Figure 2: Plots of effect size and sample size for tests of the TriversWillard hypothesis in ungulates, separated by (a) type of maternal condition measure (open circles, morphological or physiological condition; filled circles, behavioral dominance) and (b) timing of maternal condition measure relative to conception (open circles, postconception; filled circles, preconception).

sures of condition than studies of captive populations $\left(\chi^{2}=3.12, \mathrm{df}=1, P=.077, n=35\right)$, presumably because of the relative ease of collecting such data. Thus, while our analyses suggest no effect of management status on the strength of the relationship between sex ratio and maternal condition, it is possible that this could be confounded with the type of measure of condition used.

There were significant differences between different categories of studies in the sample sizes analyzed; these tended to reflect logistic constraints. Studies that had used behavioral dominance as the measure of maternal quality had smaller sample sizes than those that had used a morphological or physiological measure of condition (Wilcoxon Mann-Whitney $U$-test, $z=3.31, P=.001$; median sample sizes were 22 and 128.5 , respectively; see also fig. 2). Studies of captive populations also employed smaller sample sizes $(z=2.57, P=.01$; median for captive 33 , for wild 94.5), but there was no difference in sample size between those studied measuring condition before or after conception $(z=1.43, P=.15)$. Because meta-analysis weights effect size by sample size, the fact that the category of studies with larger sample sizes (those based on morphological measures) has a smaller mean effect size suggests that these studies will have a disproportionate effect on the overall analysis.

\section{Life-History Correlates of Sex Ratio Adjustment}

To test for potential life-history correlates of sex ratio adjustment, we first derived a mean effect size for each species by calculating a sample size weighted effect size for that species. We then performed mixed model metaanalysis on these species means, fitting either continuous or categorical life-history variables as predictor variables (see appendix $\mathrm{C}$ for details of the traits used in the analysis). These analyses suggested that degree of sexual size dimorphism $\left(b=0.275 \pm 0.381, P_{\mathrm{r}}=.026\right)$ and gestation period $\left(b=-0.00063 \pm 0.00055, P_{\mathrm{r}}=.068\right)$ were both related to the strength of the relationship between sex ratio and maternal condition. Species with more marked male-biased sexual size dimorphism tended to have stronger positive relationships between sex ratio and maternal condition; species with shorter gestation periods also had stronger positive relationships between sex ratio and maternal condition (although this relationship was only close to significance). However, because species with shorter gestation periods (at least within this data set) showed more marked male-biased sexual size dimorphism $\left(r_{17}=-0.64, P=.005\right)$, we cannot distinguish which of these two variables may be driving the relationship. In contrast, litter size $\left(P_{\mathrm{r}}=.49\right)$, mating system $\left(P_{\mathrm{r}}=.90\right)$, and social system $\left(P_{\mathrm{r}}=.15\right)$ were unrelated to the strength of the relationship between offspring sex ratio and maternal condition.

\section{Tests for Publication Bias}

We explored the possibility that publication bias had influenced the meta-analysis using first the whole data set and, second, splitting it into studies based on behavioral measures of condition and those based on morphological measures of condition. In the whole data set, the correlation between effect size and sample size was not significantly different from $0\left(r_{s}=-0.21, P=.22\right)$, and the 
calculated fail-safe number (the number of unpublished studies of zero effect needed to make the overall mean effect size not different from 0; Rosenthal 1991) was 399. For studies based on morphological measures of condition $(N=26)$, the fail-safe number was 128 , and the correlation between effect size and sample size was not significantly different from $0\left(r_{S}=-0.29, P=.16\right)$. For studies based on behavioral measures of condition $(n=11$, including Mendl et al.'s 1995), the fail-safe number was 61, and the correlation between effect size and sample size was again not significantly different from $0\left(r_{S}=0.04\right.$, $P=.91)$. Excluding Mendl et al.'s study (1995), the failsafe number for behavioral measures of condition was 80 . Finally, using all of the studies, we tested whether effect size showed any relationship with the year in which the study was published. The effect of year of publication on effect size was negative but not significantly so (slope: $\left.b=-0.0075 \pm 0.0054 \mathrm{SE}, P_{\mathrm{r}}=.10\right)$. None of these analyses provides strong evidence for a publication bias; they also suggest that the conclusion of an overall significant effect of maternal condition on the sex ratio of offspring is tolerably robust. Rosenthal (1991) proposed a rule of thumb to assess the size of fail-safe numbers that would give cause for concern as $X \leq(5 N+10)$, where $X$ is the fail-safe number and $N$ is the number of studies in the meta-analysis. By this criterion, the conclusions of a significant effect size based on all the studies combined and on behavioral measures excluding Mendl et al.'s (1995) are robust ( $X=190$ and $X=60$, respectively), while those using morphological measures of condition and behavioral measures including Mendl et al.'s 1995 are not ( $X=140$ and $X=65$, respectively), although the conclusion is marginal.

Last, we attempted to correct for any bias in publication dependent on effect size using the trim-and-fill method of Duvall and Tweedie (2000). This method estimates the number of studies that are missing from a meta-analysis as a result of any publication bias against nonsignificant results, together with their effect size, and involves adding them to the existing data set and recalculating the overall mean effect size as a test of whether a result is robust to publication bias (see Jennions and Møller 2002 for a review of this method and an assessment of publication bias in ecology). The trim-and-fill method suggested that the number of missing studies, $L_{0}$, was four, and addition of these studies reduced the estimated mean effect size for all of the studies combined to 0.071 . However, the $99 \%$ CI for this estimate (0.004-0.138) did not include 0 , suggesting that the result is robust to correction for publication bias. The change in estimated mean effect size from 0.089 to 0.071 represents a change of $20 \%$, which is less than the average change in effect size in ecological metaanalyses following this form of correction (Jennions and
Møller 2002). In summary, the analyses of publication bias do not provide strong evidence that publication bias will have influenced the effects of this meta-analysis, although they do suggest that the conclusions from the metaanalysis might be subject to reappraisal if there are a large number of unpublished studies.

\section{Discussion}

Our analyses suggest that when studies are combined, there is weak but confirmatory support for Trivers and Willard's (1973) hypothesis that maternal condition should be positively related to the sex ratio of offspring produced in ungulate mammals. However, the extent of support depends on the characteristics of the study. Studies that employed behavioral measures of dominance as the measure of maternal quality and measured these before conception supported the hypothesis more strongly than did those that employed morphological or physiological measures of condition measured after conception. In addition, Trivers-Willard effects were more likely to be positive in species with shorter gestation times and with more strongly male-biased sexual size dimorphism. Both the latter patterns are expected if the strength of sex ratio adjustment depends on maternal ability to predict the environment (West and Sheldon 2002) and on the strength of sexual selection in a species (Hewison and Gaillard 1999).

\section{Types of Maternal Quality Measure}

There are several reasons to expect a morphological or physiological measure of condition to give a poorer index of maternal ability to invest in offspring than behavioral dominance. First, in mammals, which feed continuously and reproduce many times over their lives, resources available for reproduction are likely to be determined more by continued future access than by prior access. Future access to resources would seem more likely to be related to behavioral dominance than to condition itself. Second, while some studies have shown that morphological condition and behavioral dominance are correlated (e.g., CluttonBrock et al. 1986), the correlation is considerably less than unity (e.g., $r=0.50$ for Clutton-Brock et al. 1986). If dominance is a better predictor of access to resources than morphological condition, then the expected relationship between maternal morphological condition and sex ratio will be reduced by a factor equal to the correlation between behavioral dominance and condition. Third, the relationship between a morphological measure of condition and future access to resources need not be positive. For example, in some bird species, dominant individuals carry 
lower fat reserves than do subordinate individuals (e.g., Ekman and Lilliendahl 1993). This is thought to be due to dominance status (i.e., future ability to obtain resources) influencing optimal fat reserves. If carrying large reserves of fat entails costs (e.g., as a result of impaired predator avoidance or physiological costs; Witter and Cuthill 1993), then if an individual is able to obtain food when desired as a result of being dominant at feeding sites, optimum fat reserves are lower than if the ability to obtain food is less certain. We are not aware of any studies of dominance in relation to fat reserves in mammals that support this suggestion, but it suggests that a positive relationship between fat reserves and the ability to obtain resources for offspring cannot necessarily be assumed.

A second type of explanation for a qualitative difference between data obtained using morphological measures and that obtained using behavioral dominance measures concerns the ease with which such data are obtained. As was the case for several studies in the meta-analysis, data on body size or fat reserves and the sex ratio can easily be obtained from a large sample of animals sampled over a range of environments (e.g., as a result of culling or shooting for sport). In contrast, data concerning individual variation in behavioral dominance require lengthy observations of a well-defined group of individually recognizable animals. This may be of significance for several reasons. First, some morphological measures (such as fat index or body mass) are point estimates, whereas behavioral dominance is a measure that requires repeated sampling events to obtain. All else being equal, the sampling error will be reduced for an estimate based on multiple observations. Second, combining individuals sampled from a large geographical range may combine samples that differ in their functional relationships between the variables of interest. While studies of the relationship between sex ratio and maternal condition have the advantage that they may be based on large sample sizes, the advantage of large sample size may be compromised if the data are of lower quality or contain other sources of variation that potentially confound the relationship of interest.

\section{Time of Sex Ratio Measure}

A second source of difference in effect size between studies was the timing of measurement of female condition with respect to conception. Whether maternal condition is measured pre- or postconception might have consequences for the relationship to be expected between the sex ratio and maternal condition. If developing male embryos require greater resources than do females, the sex ratio that a female carries might then influence the condition of the female. Hence, measuring maternal condition postcon- ception could confound two different processes: a positive effect of maternal condition on the sex ratio conceived and a negative effect of the sex ratio conceived on subsequent maternal condition. These processes might, in principle, counteract each other to produce anything from negative to positive relationships between the sex ratio and maternal condition depending on when, after conception, the relationships were assessed.

\section{Alternative Measures of Sex-Biased Investment}

In this meta-analysis, we have concentrated only on relationships between the sex ratio (generally measured no later than birth) and maternal condition, defined either in terms of behavioral dominance or a morphological or physiological measure of condition. There are at least two other ways in which this and related issues could be studied. If the relative return from parental investment in the two sexes depends on maternal condition, then one may also expect to see differences in the amount of parental investment that females provide toward male and female offspring dependent on maternal condition (Trivers and Willard 1973; Clutton-Brock 1991; Hewison and Gaillard 1999). We have not attempted to review the evidence for sex-biased maternal investment of this kind for a number of reasons. First, it would seem very difficult to distinguish between the effects of sex differences in demand and sex differences in investment (actually measured as maternal care). Several studies have shown that the cost of rearing different sexes of offspring may differ, with, for example, the birth of a male calf being more likely to result in a barren year in the subsequent breeding season (e.g., Clutton-Brock 1991). However, this finding simply suggests that male offspring cost more to rear than females and is not of itself evidence that sex allocation is varied with respect to maternal quality. Alternative measures of maternal investment, such as suckling frequency or milk yield rate (see Cameron 1998), are problematic because they may be determined by the sex of offspring reared, are difficult to measure satisfactorily, and may be only weakly related to maternal investment if individuals differ in how much they can expend on parental care (van Noordwijk and de Jong 1986). Whether parents of different quality adjust their relative investment in male and female offspring is a fascinating question but is one that might best be studied by experimentally redistributing offspring of different sexes among parents of different quality (e.g., Lessells et al. 1998).

Many of the studies that we surveyed here also report associations between offspring sex ratio and maternal age (e.g., Côté and Festa-Bianchet 2001). Such effects might represent Trivers-Willard-type effects if maternal condition 
or the ability to invest in offspring vary with age. In addition, if males cost more to rear than females, then selection might favor producing a relatively greater proportion of male offspring later in life as parental reproductive value declines. The latter process might also lead to agedependent sex biases in parental care, with older females willing to invest relatively more in male offspring than younger females. Saltz (2001) suggested that the data relating to associations between sex ratio and maternal age from ungulates supported a Trivers-Willard-type model. However, as pointed out by Hewison et al. (2002), the use of maternal age as a surrogate for condition is problematic because of the potentially conflicting effects of increasing condition with age (e.g., because of experience) and declining condition due to senescence.

\section{Assumptions of the Trivers-Willard Hypothesis}

As pointed out by several authors (e.g., Hewison and Gaillard 1999; Cockburn et al. 2002), a key component of the Trivers-Willard hypothesis concerns the assumptions that underlie the hypothesis. In short, these are that maternal condition influences offspring fitness, the fitness of sons is more variable than that of daughters, and sons of females in good condition will have higher fitness than daughters of females in good condition. These underlying assumptions are difficult to test, much more so than to collect data that ostensibly tests the main prediction of the TriversWillard hypothesis. In some cases, data have been collected that support all three hypotheses (e.g., Clutton-Brock et al. 1986); in other cases, the data are less clear in their support for the assumptions (for further discussion, see Hewison and Gaillard 1999). In the analyses presented here, we assumed, for simplicity, that the prediction of the Trivers-Willard hypothesis would be that females in good condition or dominant over other females should be selected to produce an excess of sons. As Leimar (1996) has shown, when reproductive value is used as the measure of offspring fitness, the direction of the effect expected depends on the way in which maternal condition is inherited. With strong maternal transmission, the expectation may even change direction. Interestingly, the single study of Cape mountain zebras included here, which had a large negative effect size $(r=-0.547)$, albeit with a low sample size, concerns a population in which maternal dominance affects only the fitness of daughters (Lloyd and Rasa 1989); in this case, the direction of the expected effect should be reversed. Changing the sign of the effect size in this study increases the estimated mean effect size for all studies to $r=+0.096$ and strengthens the conclusion for the comparison between different types of maternal quality measure $\left(\chi^{2}=13.37, \mathrm{df}=1, P_{\mathrm{r}}=.002\right)$ and differently timed measures of condition $\left(\chi^{2}=6.33\right.$, df $=1, P_{\mathrm{r}}=$ $.028)$.

\section{Conclusions}

Our analyses suggest that, when assessed under certain conditions, there is a positive relationship between offspring sex ratio and maternal quality in ungulates, as originally suggested by Trivers and Willard (1973). Does this constitute support for Trivers and Willard's model? We argue that while the data are consistent with Trivers and Willard's prediction, they do not provide a definitive test.

The first problem is that, as several authors have noted (Clutton-Brock et al. 1986; Hewison and Gaillard 1999; Forchhammer 2000), sex ratio and maternal quality may be related for reasons unrelated to Trivers and Willard's original hypothesis. A correlation might arise simply if a sex bias in mortality is correlated with maternal condition such that high-quality females are more likely to carry offspring to term. Whether this is the cause of any relationship between sex ratio and maternal condition might be assessed by determining whether the relationship is symmetrical around a mean sex ratio of 0.5 ; this was beyond the scope of this study. In some cases (e.g., CluttonBrock et al. 1984), the relationship appears symmetrical, suggesting that high-quality females may have male-biased sex ratios at implantation (see also Flint et al. 1997). In other cases (e.g., Kohlmann 1999), the relationship between sex ratio and maternal condition has an asymptote around 0.5 , which is consistent with male embryos having higher mortality than females when carried by females in poor condition. Both processes may well operate in concert, or even vary in strength between different populations, or vary in time within populations. For example, Kruuk et al. (1999) showed that a relationship between dominance and sex ratio in red deer was abolished when population density was high (see also Clutton-Brock et al. 2002). In addition, large-scale population (and possibly climatic) effects may be important determinants of variation in sex ratios at the scale of populations (Kruuk et al. 1999; Post et al. 1999; Mysterud et al. 2000; Weladji and Holand 2003). The operation of such effects may then reduce the ability to detect individual adjustment of the sex ratio.

A second argument is that formal models based on maximizing reproductive value show that the relationship between maternal quality and the sex ratio cannot be predicted without detailed life-history data applying to each case (Leimar 1996). Hence, the agreement with Trivers and Willard's original prediction that we have demonstrated in the case of ungulates may be not be true in other taxa. In particular, primates seem to present a bewildering range 
of relationships between sex ratio and maternal condition (Hiraiwa-Hasegawa 1993; Packer et al. 2000; Brown 2001; Brown and Silk 2002), and this may be because maternal effects on daughters are more prevalent. Consequently, although Trivers and Willard's original argument may explain the patterns seen in ungulates, this may have more to do with a particular coincidence of life-history traits and patterns of maternal-offspring inheritance.

\section{Acknowledgments}

We are grateful to S. Côté, A. Illius, M. Murray, C. Pélabon, and J. Perez-Barberia for providing us with data; to $\mathrm{M}$. Jennions for providing a spreadsheet to perform the trimand-fill calculations; and to two referees for comments. Our research was funded by the Biotechnology and Biological Sciences Research Council, Natural Environment Research Council, and Royal Society University Research Fellowships.

\section{Literature Cited}

Alados, C. L., and J. M. Escos. 1994. Variation in the sex ratio of a low dimorphic polygynous species with high levels of maternal reproductive effort: Cuvier's gazelle. Ethology Ecology and Evolution 6:301-311.

Arnqvist, G., and D. Wooster. 1995. Meta-analysis: synthesizing research findings in ecology and evolution. Trends in Ecology \& Evolution 10:236-240.

Austad, S. N., and M. E. Sunquist. 1986. Sex ratio manipulation in the common opossum. Nature 324:58-60.

Badyaev, A. V., G. E. Hill, M. L. Beck, A. A. Dervan, R. A. Duckworth, K. J. McGraw, P. M. Nolan, and L. A. Whittingham. 2002. Sex-biased hatching order and adaptive population divergence in a passerine bird. Science 295:316-318.

Birgersson, B. 1998. Adaptive adjustment of the sex ratio: more data and considerations from a fallow deer population. Behavioral Ecology 9:404-408.

Bourke, A. F. G., and N. R. Franks. 1995. Social evolution in ants. Princeton University Press, Princeton, N.J.

Brown, G. R. 2001. Sex-biased investment in non-human primates: can Trivers and Willard's theory be tested? Animal Behaviour 61:683-694.

Brown, G. R., and J. B. Silk. 2002. Reconsidering the null hypothesis: is maternal rank associated with birth sex ratios in primate groups? Proceedings of the National Academy of Sciences of the USA 99:11252-11255.

Burke, R. L., and J. M. Birch. 1995. White-tailed deer vary offspring sex-ratio according to maternal condition and age. Ecological Research 10:351-357.
Byers, J. A. 1997. American pronghorn: social adaptations and the ghosts of predators past. University of Chicago Press, Chicago.

Cameron, E. Z. 1998. Is suckling behaviour a useful predictor of milk intake? a review. Animal Behaviour 56: 521-532.

Cameron, E. Z., W. L. Linklater, K. J. Stafford, and C. J. Veltman. 1999. Birth sex ratios relate to mare condition at conception in Kaimanawa horses. Behavioral Ecology 10:472-475.

Cassinello, J. 1996. High-ranking females bias their investment in favour of male calves in captive Ammotragus lervia. Behavioral Ecology and Sociobiology 38:417-424.

Cassinello, J., and M. Gomendio. 1996. Adaptive variation in litter size and sex ratio at birth in a sexually dimorphic ungulate. Proceedings of the Royal Society of London B 263:1461-1466.

Chapman, N. G., M. Furlong, and S. Harris. 1997. Reproductive strategies and the influence of date of birth on growth and sexual development of an aseasonally breeding ungulate: Reeve's muntjac (Muntiacus reevesi). Journal of Zoology 241:551-570.

Charnov, E. L. 1979. The genetical evolution of patterns of sexuality: Darwinian fitness. American Naturalist 113: $465-480$.

- 1982. The theory of sex allocation. Princeton University Press, Princeton, N.J.

Charnov, E. L., R. L. Los den Hartogh, W. T. Jones, and J. van den Assem. 1981. Sex ratio evolution in a variable environment. Nature 289:27-33.

Clutton-Brock, T. H. 1991. The evolution of parental care. Princeton University Press, Princeton, N.J.

Clutton-Brock, T. H., and G. R. Iason. 1986. Sex-ratio variation in mammals. Quarterly Review of Biology 61: 339-374.

Clutton-Brock, T. H., S. D. Albon, and F. E. Guinness. 1984. Maternal dominance, breeding success, and birth sex ratios in red deer. Nature 308:358-360.

- 1986. Great expectations: dominance, breeding success and offspring sex ratios in red deer. Animal Behaviour 34:460-471.

Clutton-Brock, T. H., T. N. Coulson, E. J. Milner-Gulland, D. Thomson, and H. M. Armstrong. 2002. Sex differences in emigration and mortality affect optimal management of deer populations. Nature 415:633-637.

Cockburn, A., S. Legge, and M. C. Double. 2002. Sex ratios in birds and mammals: can the hypotheses be disentangled? Pages 266-286 in I. C. W. Hardy, ed. Sex ratios: concepts and research methods. Cambridge University Press, Cambridge.

Côté, S. D., and M. Festa-Bianchet. 2001. Offspring sex ratio in relation to maternal age and social rank in 
mountain goats (Oreamnos americanus). Behavioral Ecology and Sociobiology 49:260-265.

Duvall, S., and R. Tweedie. 2000. Trim and fill: a simple funnel-plot-based method of testing and adjusting for publication bias in meta-analysis. Biometrics 56:455463.

Ekman, J. B., and K. Lilliendahl. 1993. Using priority to food access: fattening strategies in dominance-structured willow tit (Parus montanus) flocks. Behavioral Ecology 4:232-238.

Fernandez-Llario, P., J. Carranza, and P. Mateos-Quesada. 1999. Sex allocation in a polygynous mammal with large litters: the wild boar. Animal Behaviour 58:1079-1084.

Festa-Bianchet, M. 1991. The social system of bighorn sheep: grouping patterns, kinship and female dominance rank. Animal Behaviour 42:71-82.

- 1996. Offspring sex ratio in studies of mammals: does publication depend upon the quality of the research or the direction of the results? Ecoscience 3:4244.

Flint, A. P. F., S. D. Albon, and S. I. Jafar. 1997. Blastocyst development and conceptus sex selection in red deer Cervus elaphus: studies of a free-living population on the Isle of Rum. General and Comparative Endocrinology 106:374-383.

Forchhammer, M. C. 2000. Timing of foetal growth spurts can explain sex ratio variation in polygynous mammals. Ecology Letters 3:1-4.

Frank, S. A. 1990. Sex allocation theory for birds and mammals. Annual Review of Ecology and Systematics 21:13-55.

- 1998. Foundations of social evolution. Princeton University Press, Princeton, N.J.

Godfray, H. C. J. 1994. Parasitoids. Princeton University Press, Princeton, N.J.

Gurevitch, J., and L. V. Hedges. 1999. Statistical issues in ecological meta-analyses. Ecology 80:1142-1149.

Hass, C. C. 1991. Social status in female bighorn sheep (Ovis canadensis): expression, development and reproductive correlates. Journal of Zoology 225:509-523.

Hewison, A. J. M., and J.-M. Gaillard. 1996. Birth sex ratios and local resource competition in roe deer, Capreolus capreolus. Behavioral Ecology 7:461-464.

- 1999. Successful sons or advantaged daughters? the Trivers-Willard model and sex-biased investment in ungulates. Trends in Ecology \& Evolution 14:229-234.

Hewison, A. J. M., R. Andersen, J.-M. Gaillard, J. D. C. Linnell, and D. Delorme. 1999. Contradictory findings in studies of sex ratio variation in roe deer (Capreolus capreolus). Behavioral Ecology and Sociobiology 45:339348.

Hewison, A. J. M., J.-M. Gaillard, P. Blanchard, and M. Festa-Bianchet. 2002. Maternal age is not a predominant determinant of progeny sex ratio variation in ungulates. Oikos 98:334-339.

Hiraiwa-Hasegawa, M. 1993. Skewed sex ratios in primates: should high-ranking mothers have daughters or sons? Trends in Ecology \& Evolution 8:395-400.

Huck, U. W., J. Seger, and R. D. Lisk. 1990. Litter sex ratios in the golden hamster vary with time of mating and are not binomially distributed. Behavioral Ecology and Sociobiology 26:99-109.

Jennions, M. D., and A. P. Møller. 2002. Publication bias in ecology and evolution: an empirical assessment using the "trim and fill" method. Biological Reviews 77:211222.

Kohlmann, S. G. 1999. Adaptive fetal sex allocation in the elk: evidence and implications. Journal of Wildlife Management 63:1109-1117.

Kojola, I. 1993. Early maternal investment and growth in reindeer. Canadian Journal of Zoology 71:753-758.

. 1997a. Social status and physical condition of mother and sex ratio of offspring in cervids. Applied Animal Behaviour Science 51:267-274.

Kojola, I., and E. Eloranta. 1989. Influences of maternal body weight, age and parity on sex ratio in semidomesticated reindeer. Evolution 43:1331-1336.

Kojola, I., and T. Helle. 1994. Offspring sex ratio adjustment in reindeer, Rangifer tarandus. Annales Zoologici Fennici 31:405-410.

Krackow, S., E. Meelis, and I. C. W. Hardy. 2002. Analysis of sex ratio sequences and sequences of sex allocation. Pages 112-131 in I. C. W. Hardy, ed. Sex ratios: concepts and research methods. Cambridge University Press, Cambridge.

Kruuk, L. E. B., T. H. Clutton-Brock, S. D. Albon, J. M. Pemberton, and F. E. Guinness. 1999. Population density affects sex ratio variation in red deer. Nature 399: 459-461.

Leimar, O. 1996. Life-history analysis of the Trivers and Willard problem. Behavioral Ecology 7:316-325.

Lessells, C. M., K. R. Oddie, and A. C. Mateman. 1998. Parental behaviour is unrelated to experimentally manipulated great tit brood sex ratio. Animal Behaviour 56:385-393.

Lindström, J., T. Coulson, L. Kruuk, M. C. Forchhammer, D. W. Coltman, and T. Clutton-Brock. 2002. Sex-ratio variation in Soay sheep. Behavioral Ecology and Sociobiology 53:25-30.

Llandete-Castillejos, T., A. Garcia, S. Langton, I. Inglis, L. Gallego, and J. Garde. 2001. Opposing offspring sex ratio variations with increasing age and weight in mouflon mothers. Acta Veterinaria Hungarica 49:257-268.

Lloyd, P. H., and O. A. E. Rasa. 1989. Status, reproductive success and fitness in Cape mountain zebra (Equus zebra 
zebra). Behavioral Ecology and Sociobiology 25:411420.

Meikle, D. B., L. C. Drickamer, S. H. Vessey, T. L. Rosenthal, and K. S. Fitzgerald. 1993. Maternal dominance rank and secondary sex ratio in domestic swine. Animal Behaviour 46:79-85.

Meikle, D. B., L. C. Drickamer, S. H. Vessey, R. D. Arthur, and T. L. Rosenthal. 1996. Dominance rank and parental investment in swine (Sus scrofa domesticus). Ethology 102:969-978.

Meikle, D. B., S. H. Vessey, and L. C. Drickamer. 1997. Testing models of adaptive adjustment of secondary sex ratio in domestic swine. Animal Behaviour 53:428-431.

Mendl, M., A. J. Zanella, D. M. Broom, and C. T. Whittemore. 1995. Maternal social status and birth sex ratio in domestic pigs: an analysis of mechanisms. Animal Behaviour 50:1361-1370.

- 1997. Studying birth sex ratio in domestic pigs. Animal Behaviour 53:432-435.

Monard, A. M., P. Duncan, H. Fritz, and C. Feh. 1997. Variations in the birth sex ratio and neonatal mortality in a natural herd of horses. Behavioral Ecology and Sociobiology 41:243-249.

Mysterud, A., N. G. Yoccoz, N. C. Stenseth, and R. Langvatn. 2000. Relationships between sex ratio, climate and density in red deer: the importance of spatial scale. Journal of Animal Ecology 69:959-974.

Nygén, T., and I. Kojola. 1997. Twinning and fetal sex ratio in moose: effects of maternal age and mass. Canadian Journal of Zoology 75:1945-1948.

Packer, C., D. A. Collins, and L. E. Eberley. 2000. Problems with primate sex ratios. Philosophical Transactions of the Royal Society of London B 355:1627-1635.

Palmer, A. R. 2000. Quasi-replication and the contract of error: lessons from sex ratios, heritabilities and fluctuating asymmetry. Annual Review of Ecology and Systematics 31:441-480.

Post, E., M. C. Forchhammer, N. C. Stenseth, and R. Langvatn. 1999. Extrinsic modification of vertebrate sex ratios by climatic variation. American Naturalist 154:194204.

Ranta, E., A. Laurila, and J. Elmberg. 1994. Reinventing the wheel: analysis of sexual dimorphism in body size. Oikos 70:313-321.

Reimers, E. 1999. Foetal sex ratios in wild reindeer Rangifer tarandus in relation to maternal condition and age. Wildlife Biology 5:49-54.

Reimers, E., and D. Lenvik. 1997. Fetal sex ratio in relation to maternal mass and age in reindeer. Canadian Journal of Zoology 75:648-650.

Rice, W. R., and S. D. Gaines. 1994. Extending nondirectional heterogeneity tests to evaluate simply or- dered alternative hypotheses. Proceedings of the $\mathrm{Na}$ tional Academy of Sciences of the USA 91:225-226.

Rosenberg, M. S., D. C. Adams, and J. Gurevitch. 2000. MetaWin: statistical software for meta-analysis. Version 2.0. Sinauer, Sunderland, Mass.

Rosenthal, R. 1991. Meta-analytic procedures for social research. Sage, Beverly Hills, Calif.

Rutberg, A. T. 1986. Lactation and foetal sex ratios in American bison. American Naturalist 127:89-94.

Saltz, D. 2001. Progeny sex ratio variation in ungulates: maternal age meets environmental perturbation of demography. Oikos 94:377-384.

Schwartz, C. C., and K. J. Hundertmark. 1993. Reproductive characteristics of Alaskan moose. Journal of Wildlife Management 57:454-468.

Shaw, J. H., and T. S. Carter. 1989. Calving patterns among American bison. Journal of Wildlife Management 53: 896-898.

Skogland, T. 1986. Sex ratio variation in relation to maternal condition and parental investment in wild reindeer. Oikos 46:417-419.

Smith, B. L., R. L. Robbins, and S. H. Anderson. 1996. Adaptive sex ratios: another example? Journal of Mammalogy 77:818-825.

Trivers, R. L., and D. E. Willard. 1973. Natural selection on parental ability to vary the sex ratio of offspring. Science 179:90-92.

van Noordwijk, A. J., and G. de Jong. 1986. Acquisition and allocation of resources: their influence on variation in life-history tactics. American Naturalist 128:137-142.

Verme, L. J. 1983. Sex ratio variation in Odocoileus: a critical review. Journal of Wildlife Management 47:573582.

- 1985. Progeny sex ratio relationships in deer: theoretical vs. observed. Journal of Wildlife Management 49:134-136.

Wauters, L. A., S. A. De Crombrugghe, N. Nour, and E. Matthysen. 1995. Do female roe deer in good condition produce more sons than daughters? Behavioral Ecology and Sociobiology 37:189-193.

Weladji, R. B., and O. Holand. 2003. Sex ratio variation in reindeer: a test of the extrinsic modification hypothesis. Wildlife Biology 9:29-36.

Weladji, R. B., O. Holand, G. Steinheim, and D. Lenvik. 2003. Sex-specific pre-weaning maternal care in reindeer (Rangifer tarandus). Behavioral Ecology and Sociobiology 53:308-314.

West, S. A., and B. C. Sheldon. 2002. Constraints in the evolution of facultative sex ratio adjustment. Science 295:1685-1688.

West, S. A., E. A. Herre, and B. C. Sheldon. 2000. The benefits of allocating sex. Science 290:288-290.

Williams, G. C. 1979. The question of adaptive sex ratio 
in out-crossed vertebrates. Proceedings of the Royal Society of London B 205:567-580.

Witter, M. S., and I. C. Cuthill. 1993. The ecological costs of avian fat storage. Philosophical Transactions of the Royal Society of London B 340:73-92.

Wolff, J. O. 1988. Maternal investment and sex ratio ad- justment in American bison calves. Behavioural Ecology and Sociobiology 23:127-133.

1998. Breeding strategies, mate choice, and reproductive success in American bison. Oikos 83:529544.

Associate Editor: Curt Lively 